\title{
Writing a multiple-choice test question
}

\author{
MATTHEW A. TERRY, DO
}

This volume of the JAOA starts a new monthly section-"Program Director's Notebook." Designed to provide practical tips for directors of medical education and residency program directors, this section will help to enhance the quality of administration, teaching, and evaluation in graduate medical education. The first article in this series addresses a common problem: how to write a multiple-choice test question.

Future articles in this series will cover topics in curriculum design, faculty development, program administration, and educational evaluation. This section should be of interest to all those individuals in teaching roles in both undergraduate and graduate osteopathic medical education. Interns and residents will also find this section useful as they prepare for careers in academic medicine.

HELEN H. BAKER, PhD

Section Editor

Multiple-choice test questions are the most widely used and highly regarded of the presently available objective or selective test items. They can be used to test all levels of learning and are applicable to the measurement of most important educational outcomes. Although it is difficult to construct these questions well, they are versatile and can be used in settings involving large numbers of students.

(Key words: Multiple-choice question, objective/selective item)

The need to assess education and to measure achievement is everpresent. Educators and scholars have been struggling for thousands of years with the question of how to best ac-

\footnotetext{
Dr Terry is Dean of the Southeastern University of the Health Sciences College of Osteopathic Medicine.

Reprint requests to Matthew A. Terry, DO, $1750 \mathrm{NE}$ 167 th St, North Miami Beach, FL 33162-03017.
}

complish this assessment. Oral examinations were probably given before human beings developed written communication. Essay tests date back to earlier than $2300 \mathrm{BC}$ in ancient China and, until the turn of the century, they were about the only form of written examination in wide use. ${ }^{1}$

Many factors, both philosophic and practical, have contributed to the development of new testing formats. In addition to the essay test, currently available formats include shortanswer, matching, true-false, and multiplechoice examinations. This article will focus on the widely popular multiple-choice-type item, which, in many settings, is used exclusively.

\section{Approaching the evaluation process}

Although a detailed discussion of the evaluation process as a whole is beyond the scope of this paper, as an educator, a few general comments should be made. First, approach the 
evaluation process the same way you approach the educational process: Decide what is important and then focus on it. Although it is acceptable to assign students some material to learn on their own, you should, for the most part, examine students on what has been taught to them. Borrowing from the taxonomy developed by Benjamin S. Bloom, ${ }^{2}$ we in medical education tend to operate on several levels of learning. These levels are knowledge, the simple recall of material; comprehension, restating material to show understanding; and application, problem-solving or applying ideas in new situations.

Your examination should reflect not only the topics covered, but also the various levels at which they were taught. A two-way grid (Figure), sometimes called a table of specifications, is a good way to organize a written plan for determining how to apportion your test content.

\section{Grouping test items}

Having defined what we are going to test, we must now determine how we are going to test it. Although many educators classify examinations as subjective or objective, I prefer to group test items into one of two categories: selection items, those such as matching, truefalse, or multiple choice, which require that students select the correct answer; and production items, which are short-answer or essay questions that require students to produce an acceptable answer. Although all these formats have a place in evaluation, multiple-choice test items are currently the most highly regarded and widely used. They are adaptable to the measurement of most important educational outcomes of knowledge, understanding, and judgment; of ability to solve problems, to recommend appropriate actions, and to make predictions.

Almost any understanding or ability that can be tested by means of any other item form can also be tested by means of the multiplechoice test item. Its strengths, like the other items in the selection category, are that they require relatively little student time to answer, they cover more of the material because more

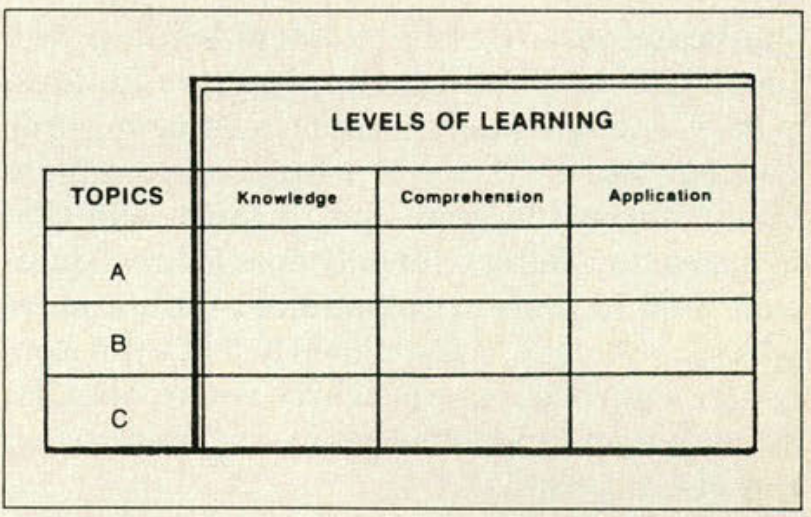

Figure. On this two-way grid, plot topics versus levels of learning and fill in each cell with the percentage of time. This same percentage devoted to the instruction should be allotted to the test.

questions can be asked, and they are easily and accurately scored by a person or machine.

Limitations also exist. Multiple-choice questions do not allow for the demonstration of knowledge beyond the range of the options provided. They are also difficult and time-consuming to write well, although many inexperienced test writers would think the opposite. Finally, it has been said that they encourage quessing, but I have never thought this to be a serious problem. (If you were, for example, to guess blindly on ten multiple-choice questions, each with five distracters, your chances of achieving a perfect score would be one in 10 million!)

\section{Anatomy of a multiple-choice question}

In considering the anatomy of a multiplechoice question item, we can dissect it into three parts: the stem, a question or incomplete sentence that clearly implies a question; the correct answer; and distracters, several plausible but incorrect answers to the question. It should also be mentioned that there are several variations of the multiple-choice question, the K-type question (that in which there are multiple combinations of correct responses within each question) being the most notorious. Here, however, we will focus on the standard A-type question, which has four or five distracters, just one of which is correct.

The function of the item stem is to acquaint 
the examinee with the problem being posed. Ideally, it should state or imply a specific question. Although one can sometimes save words without loss of clarity by using an incomplete statement as the item stem, a direct question is most often better. Not only does a direct question tend to present the student with a more specific problem, it also tends to focus the item writer's purposes more clearly and to help the student avoid irrelevance or unrelatedness in the distracters. ${ }^{3}$

One should include as much of the item as possible in the stem so that it is not necessary to repeat the same phrase in each of the options. A positive question should be used wherever possible. If a negative question must be used, the negative word should be underlined. In testing definitions, it is best to use the term being defined in the stem and to offer optional definitions as distracters. Wordy and needlessly complex stems should be avoided. Similarly, informational preambles that serve only as window dressing and do not help the examinee understand the question being asked are unnecessary and should also be avoided.

There is no set number of possible responses, although it is usually recommended that there be one correct answer and three or four distracters. On most board examinations, it has become standard to use five possible responses. The response options should be lettered using capital letters because small, or lowercase, letters can be difficult to distinguish.

When devising distracters, all options should be plausible responses. One should not write poor alternatives just for the sake of having more responses. All options should be clearly distinguished by being listed on separate lines. Preferably, the options should be somehow related-sound, content, et ceteraand should be gramatically consistent with the stem. The options for an individual item should be approximately the same length; one should especially avoid making correct responses consistently longer throughout the examination.

Ambiguous, imprecise terms such as "several," "seldom," and "frequently" should be avoided. Instead, more specific phrases such as " $50 \%$ of the time" or "two out of three" should be used. Contrarily, options that are too similar in value $(1 \%, 2 \%, 3 \%, 4 \%$, and $5 \%$ ) or artificially precise $(55.97 \%)$ should also be avoided. Whenever responses form a quantitative or qualitative scale, they normally should be arranged in order of magnitude either from smallest to largest or from largest to smallest.

A frequently used technique, but one to be avoided, is the listing of several factual responses along with other options, such as "all of the above," "none of the above," or "A and B but not C." This technique is probably most often used when the item writer can think of only a few good distracters. It should be frowned on because it tends to make the item either ambiguous or selection of the correct response too easy.

\section{Other considerations}

Aside from the details of the items themselves, there are other questions to be considered. For example, how many items should be on the test? Although, for the most part, longer examinations have increased reliability and validity, test length is most often determined by how much time is available. A good rule of thumb is to allow approximately 1 minute for each multiple-choice item. ${ }^{4}$ It is statistically convenient, then, for a 1-hour test to contain 50 items and a 2 -hour test to contain 100 items.

Another problem to deal with is the level and distribution of difficulty of the items. Without going into a lengthy or statistical discussion, theoretical analyses and experimental studies have demonstrated quite convincingly that in most situations questions that are neither very difficult nor very easy are best. ${ }^{5}$ In other words, questions of moderate difficulty will tend to "spread out" your sample and identify which of the students have mastered the material and which have not.

\section{Comment}

Much more could be said, and indeed, many chapters have been written on this subject. As stated, the multiple-choice question has, for

(continued on page 123) 


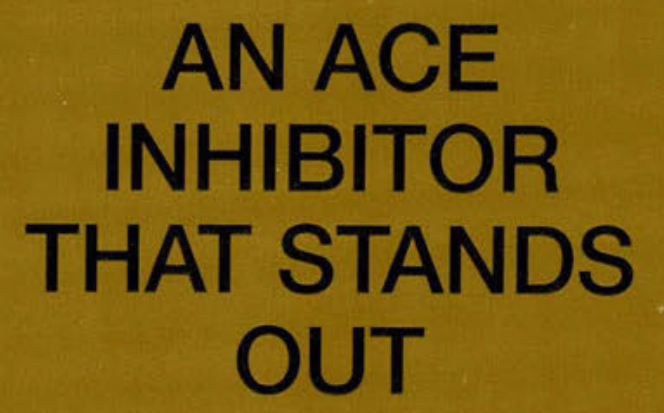



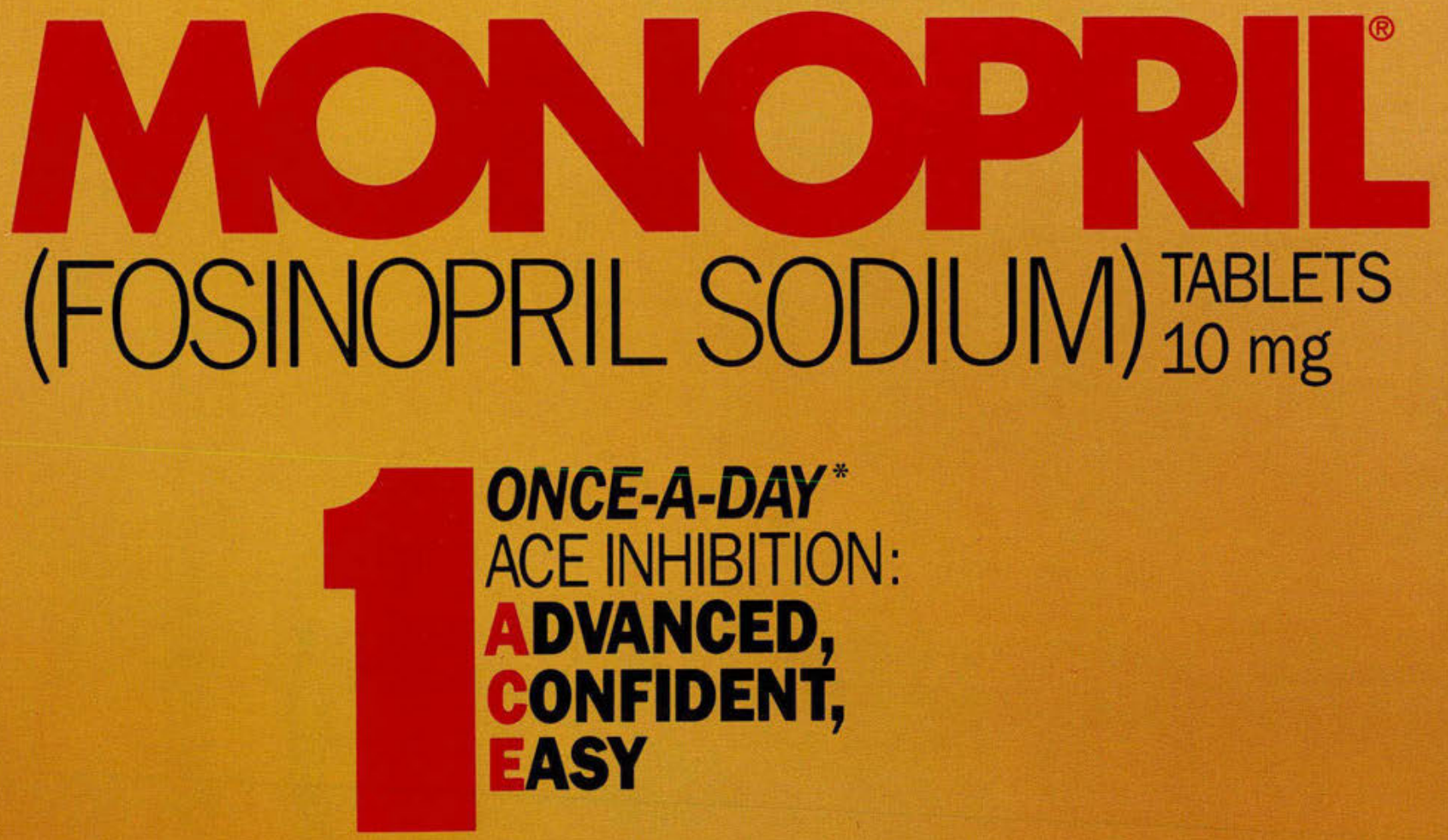

*In some patients the antihypertensive effect may

diminish toward the end of the dosing interval. If trough

response is inadequate, dividing the daily dose

should be considered. 


\section{ADVANCED ELIMINATION DUAL, BALANCED, AND COMPENSATORY}

The ACE inhibitor ${ }^{*}$ with unique dual/balanced routes of ellmination ${ }^{5}$

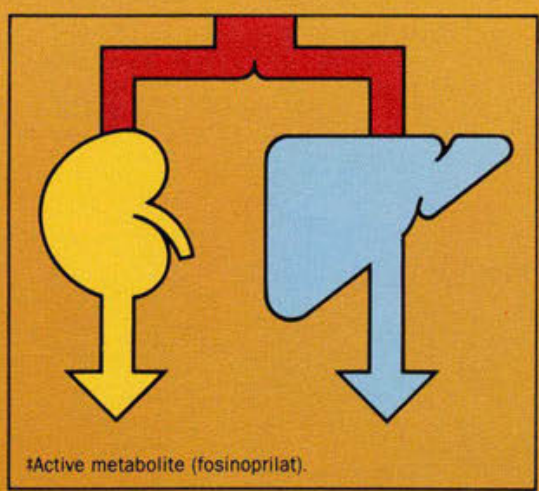

- Evaluation of the hypertensive patient should always include assessment of renal function
The ACE Inhibitor with

compensatory elimination ${ }^{\dagger}$

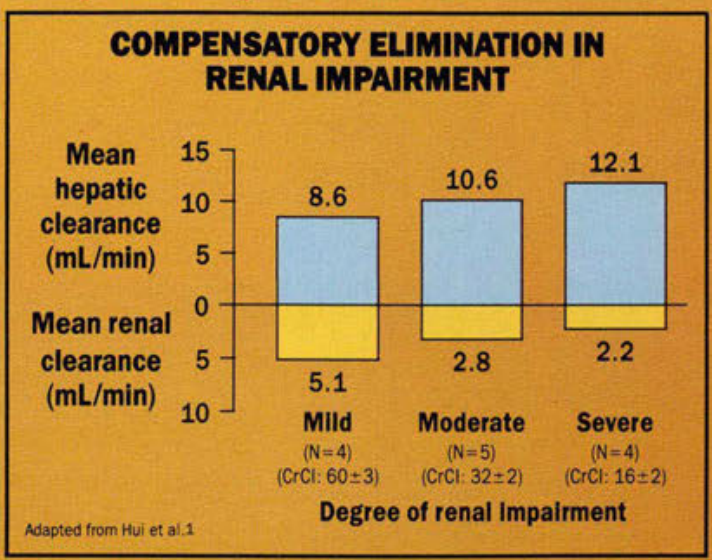

In this pharmacokinetic study, 13 evaluable patients with renal impairment received a single IV dose of $7.5 \mathrm{mg}$ radiolabeled ${ }^{14} \mathrm{C}$-fosinoprilat (equimolar to $10 \mathrm{mg}$ fosinopril sodium). A modest increase in plasma AUC levels (less than two times that in normals) was observed in patients with various degrees of renal insufficiency.

- Elimination shifts to hepatic route from mild to severe renal impairment ${ }^{1}$

\section{Minimal drug accumulation ${ }^{2}$}

†In patients with renal impairment.

$\S$ In subjects with normal renal function.

In patients with severe congestive heart failure, unilateral or bilateral renal artery stenosis, pre-existing renal disease, or on concomitant diuretics, changes in renal function may occur. In these patients, renal function should be monitored during the first few weeks of therapy. See PRECAUTIONS in the brief summary of full prescribing information on last page of this advertisement. 

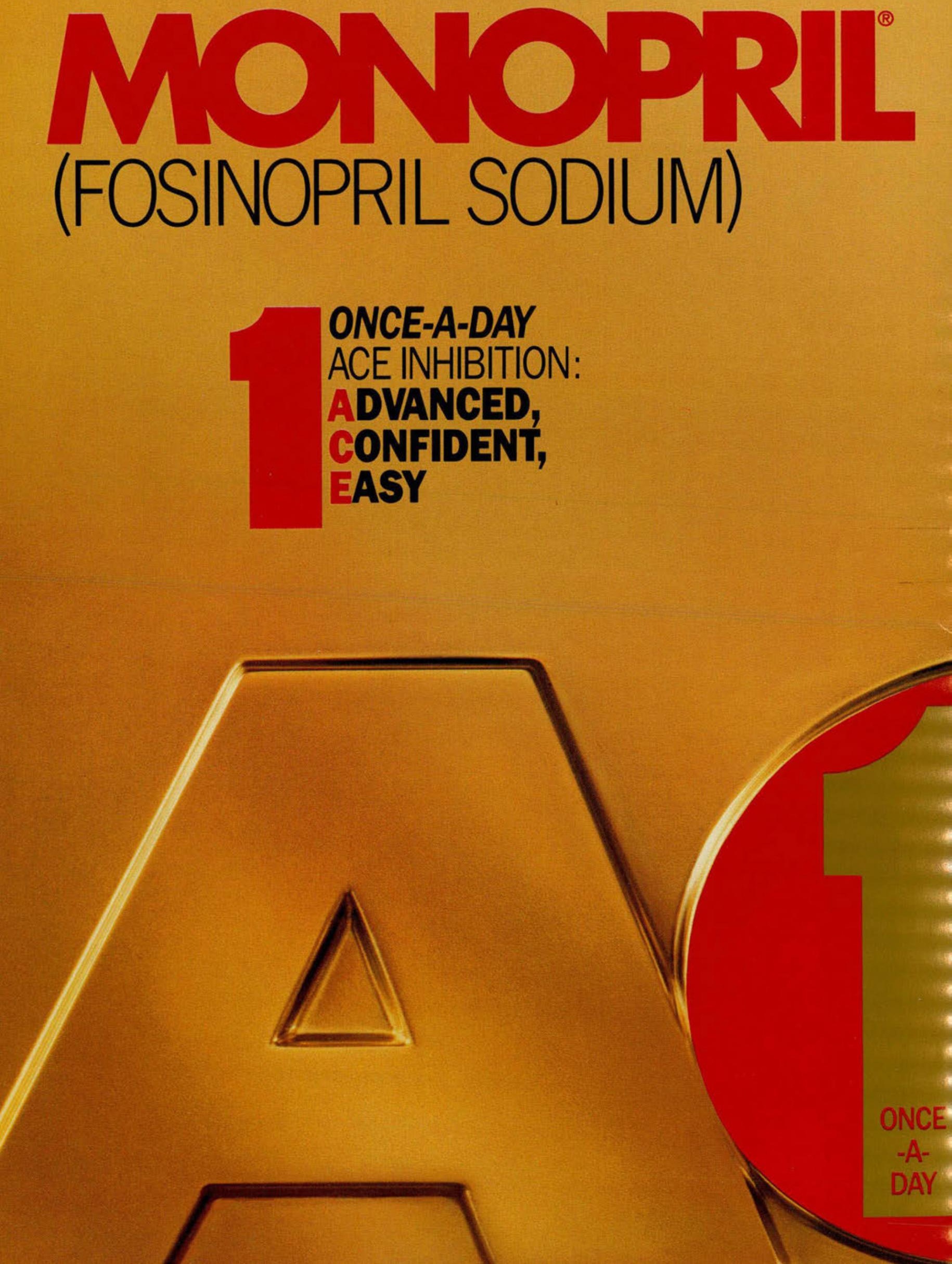


\section{CONFIDENT CONTROL FOR ABROAD RANGE OF HYPERTENSIVE PATIENTS}

\section{Long-term blood pressure control}

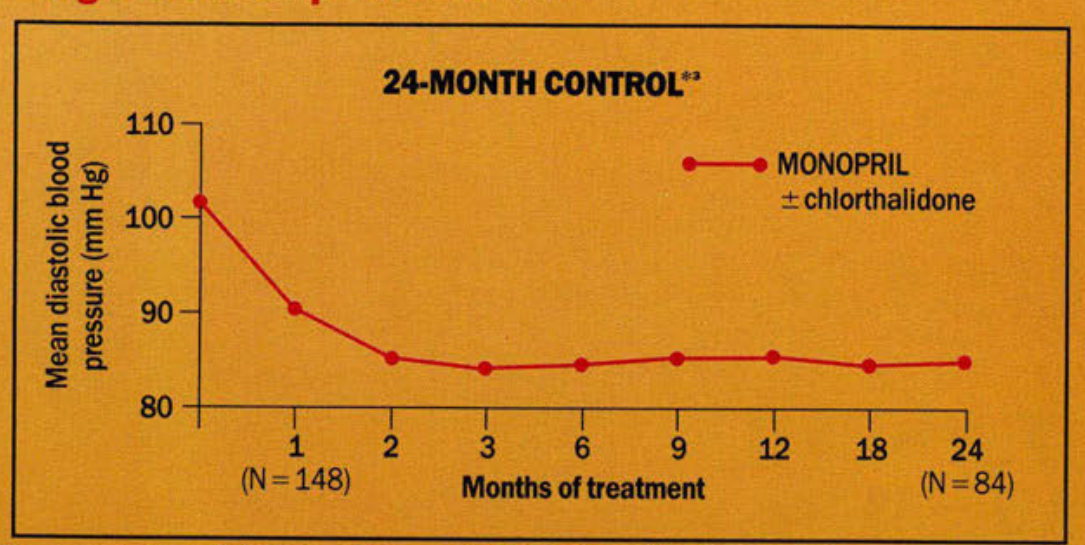

*Dosage: fosinopril 10-80 mg QD \pm up to $25 \mathrm{mg} /$ day chlorthalidone after week 4 .

Long-term, open label, multicenter, follow-up study of patients who had completed 8-week, double-blind, randomized trial of MONOPRIL 10-80 mg QD. Approximately half of patients had diuretic added.
Effective therapeutic response In older and youngert patlents, black ${ }^{*}$ and nonblack patlents

tSafety and effectiveness in individuals less than 18 years old have not been established.

¥MONOPRIL has an antihypertensive effect even in patients with low-renin hypertension. Although MONOPRIL was antihypertensive in all races studied, black hypertensive patients (usually a low-renin hypertensive population) had smaller average response to ACE inhibitor monotherapy than nonblacks.

Please see brief summary of full prescribing information on last page of this advertisement.

\section{- No attenuation of blood-pressure response over a 24-month treatment period ${ }^{3}$}



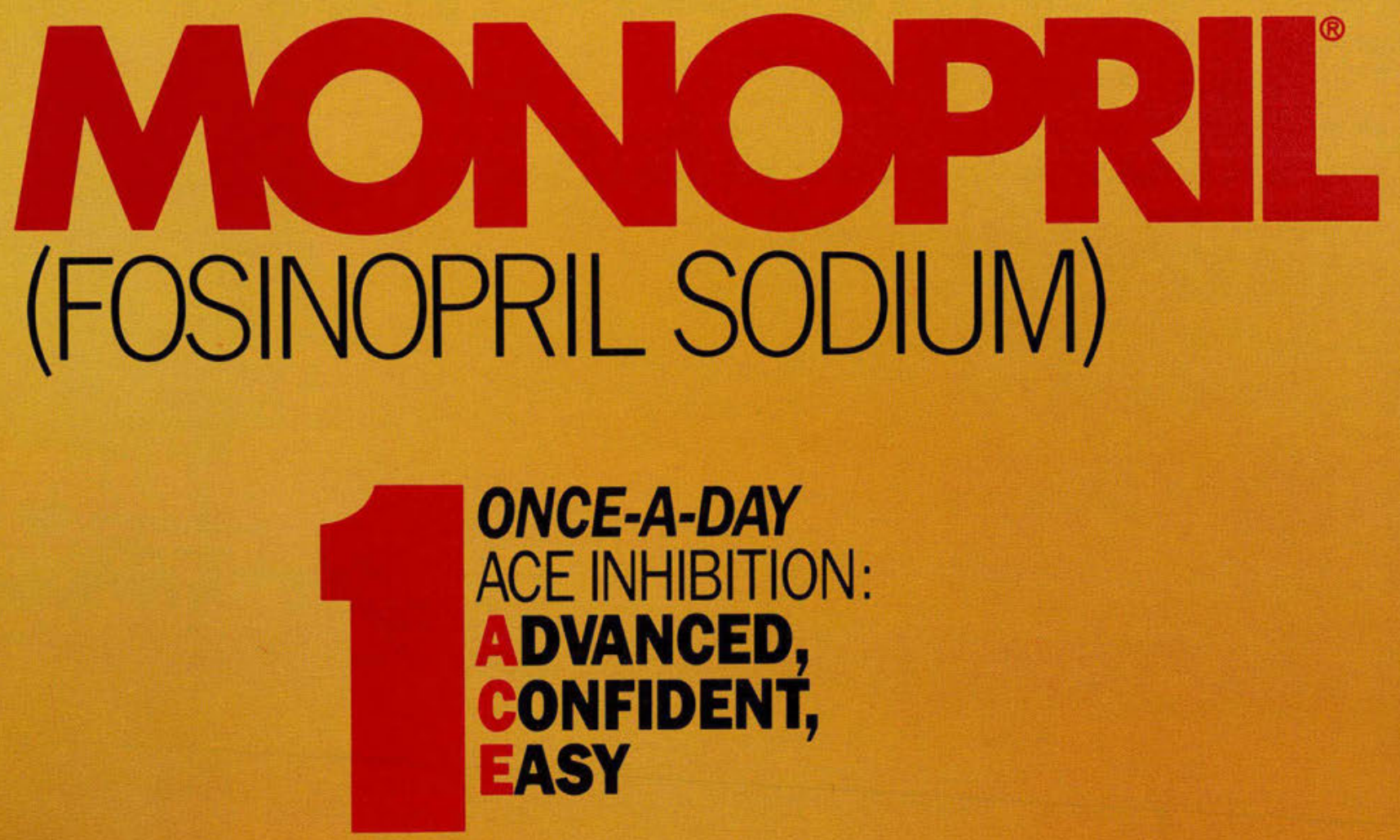


\section{EASY ADMINISTRATION 10-mg QD STARTING DOSE FOR VIRTUALLY ALL HYPERTENSIVES}

The same 10-mg QD starting dose regardless of age; race, or renal function

- Usual dosage range: 20 to $40 \mathrm{mg} \mathrm{QD}^{+}$
Excellent tolerability may enhance compliance

In placebo-controlled trials:

- Low total discontinuation rate: $4.1 \%$

- Most common side effects attributed to drug therapy (\%): headache (3.2), cough (2.2), dizziness (1.6), fatigue (1.5), diarrhea (1.5)

- Equally well tolerated in older and younger patients, ${ }^{3}$ black and nonblack patients
* Safety and effectiveness in individuals less than 18 years old have not been established.

+ In some patients the antihypertensive effect may diminish toward the end of the dosing interval. If trough response is inadequate, dividing the daily dose should be considered. Some patients appear to have a further response to $80 \mathrm{mg}$.

Please see brief summary of full prescribing information on last page of this advertisement. 
MONOPRIL Fosinopril Sodium Tablets

CONTRAINDICATIONS MONOPRIL. is contraindicated in patients who are hypersensitive to this product or to any other angiotensin converting enzyme inhibitor (e.g., a patient who has experienced angioedema with any other ACE inhibitor therapy). WARNINGS. Angioedema: Angioedema involving the extremities, face, lipS. mucous membranes, tongue, glottis or larynx has been reported in patients treated with ACE inhifilors. If or angioedema of the face, lips, mucous membranes, tongue, glottis or extremities occurs, treatment with ment of the tongue, glottis, or larynx, likely to cause airway obstruction, appropriate therapy, e.g. ment of the tongue, glottis, or larynx, likely to cause airway obstruction, appropriate therapy, e.g. subcutaneous epinephrine solution 1:1000 (0.3 mL to $0.5 \mathrm{~mL}$ ) should be promptly administered (see
PRECAUTIONS: Information for Patients and ADVERSE REACTIONS). Hypotension: MONOPRIL can cause PRECAUTIONS: Information for Patients and ADVERSE REACTIONS). Hypotension: MONOPRIL can cause
symptomatic hypotension. Like other ACE inhibitors, fosinopril has been only rarely associated with hypotension in uncomplicated hypertensive patients. Symptomatic hypotension is most likely to occur in patients who have been volume- and/or salt-depleted as a result of prolonged diuretic therapy, dietary salt restriction, dialysis, diarrhea, or vomiting. Volume and/or salt depletion should be corrected before initiating therapy with
MONOPRIL. In patients with congestive heart failure, with or without associated renal insufficiency. ACE inhibitor therapy may cause excessive hypotension, which may be associated with oliguria or azotemia and, rarely, with acute renal failure and death. In such patients, MONOPRIL. therapy should be started under close medical supervision; they should be followed closely for the first 2 weeks of treatment and whenever the dose of fosinopril or diuretic is increased. If hypotension occurs, the patient should be placed in a supine position, and if necessary, treated with intravenous infusion of physiological saline. MONOPRIL treatment usually can be
continued following restoration of blood pressure and volume. Neutropenia/Agranulocytosis: Another ancontinued following restoration of blood pressure and volume. Neutropenia/Agranulocytosis: Another an-
giotensin converting enzyme inhibitor, captopril, has been shown to cause agranulocytosis and bone marrow depression, rarely in uncomplicated patients, but more frequently in patients with renal impairment, especially if they also have a collagen-vascular disease such as systemic lupus erythematosus or scleroderma. Available data from clinical trials of fosinopril are insufficient to show that fosinopril does not cause agranulocytosis at similar rates. Monitoring of white blood cell counts should be considered in patients with collagen-vascular disease. ACE inhibitors can cause fetal and neonatal morbidity and mortality when administered to pregnant women. When ACE inhibitors have been used during the second and third trimesters of pregnancy, there have been reports of neonatal hypotension, renal failure, skull hypoplasia, and death. Oligohydramnios has also been reported, presumably resulting from decreased fetal renal function; oligohydramnios has been associated with fetal limb contractures, craniofacial malformations, hypoplastic lung development, and intrauterine growth fetal limb contractures, craniofacial malformations, hypoplastic lung development, and intrauterine growth
retardation. Prematurity and patent ductus arteriosus have been reported, although it is not clear whether these retardation. Prematurity and patent ductus arteriosus have been reported, although it is not clear whether these
occurrences were due to the ACE inhibitor exposure or to the mother's underlying disease. It is not known whether exposure limited to the first trimester can adversely affect fetal outcome. A patient who becomes pregnant while taking ACE inhibitors, or who takes ACE inhibitors when already pregnant, should be apprised of the potential hazard to her fetus. If she continues to receive ACE inhibitors during the second or third trimester of pregnancy, frequent ultrasound examinations should be performed to look for oligohydramnios. When oligohydramnios is found, ACE inhibitors should generally be discontinued.Infants with histories of in utero occurs, attention should be directed toward support of blood pressure and renal perfusion. Fosinopril is poorly dialyzed from the circulation of adults by hemodialysis and peritoneal dialysis. There is no experience with any procedure for removing fosinopril from the neonatal circulation.In pregnant rabbits, maternal toxicity was procedure for removing losinopril from the neonatal circulation.in pregnant rabbits, maternal toxicity was evident at doses ranging from 2.5 to $40 \mathrm{mg} / \mathrm{kg} /$ day (approximately 3 to 50 times the maximum recommended
human dose). Fosinopril was embryocidal in rabbits at 10 and $40 \mathrm{mg} / \mathrm{kg} /$ day (approximately 12 and 50 times the human dose). Fosinopril was embryocidal in rabbits at 10 and $40 \mathrm{mg} / \mathrm{kg} /$ day (approximately 12 and 50 times the maximum recommended human dose). These effects were probably due to marked decreases in blood pressure pregnant rats, there was evidence of maternal toxicity at all dose levels tested, i.e., 25 to $400 \mathrm{mg} / \mathrm{kg} /$ day (about 30 to 500 times the maximum recommended human dose). Slight reductions in placental weights and degree of skeletal ossification were observed at all dose levels, and fetal body weights were reduced in the high-dose group. Three similar orofacial malformations and one fetus with situs inversus occurred in fosinopri-treated animais. The association of these anomalies with treatment is uncertain. PRECAUTIONS. General: Impaired Renal Function: As a consequence of inhibiting the renin-angiotensin-aldosterone system, changes in renal
function may be anticipated in susceptible individuals. In patients with severe congestive heart failure whose function may be anticipated in susceptible individuals. In patients with severe congestive heart fallure whose renal function may depend on the activity of the renin-angiotensin-aldosterone system, treatment with
angiotensin converting enzyme inhibitors, including MONOPRIL (Fosinopril Sodium), may be associated with angiotensin converting enzyme inhibitors, including MONOPRIL (Fosinopril Sodium), may be associated with
oliguria and/or progressive azotemia and (rarely) with acute renal failure and/or death.ln hypertensive patients with renal artery stenosis in a solitary kidney or bilateral renal artery stenosis, increases in blood urea nitrogen and serum creatinine may occur. Experience with another angiotensin converting enzyme inhibitor suggests tha these increases are usually reversible upon discontinuation of ACE inhibitor and/or diuretic therapy. In such patients, renal function should be monitored during the first few weeks of therapy. Some hypertensive patients with no apparent pre-existing renal vascular disease have developed increases in blood urea nitrogen and serum creatinine, usually minor and transient, especially when MONOPRIL has been given concomitantly with a diuretic. This is more likely to occur in patients with pre-existing renal impairment. Dosage reduction of
MONOPRIL and/or discontinuation of the diuretic may be required. Evaluation of the hypertensive patient should always include assessment of renal function (see DOSAGE AND ADMINISTRATION). Impaired renal should always include assessment of renal function (see DOSAGE AND ADMINISTRATION), Impaired rena function decreases total clearance of fosinoprilat and approximately doubles AUC. In general, however, no
adjustment of dosing is needed (see CLINICAL PHARMACOLOGY). Hyperkalemia: In clinical trials, hyperkalemia (serum potassium greater than $10 \%$ above the upper limit of normal) has occurred in approximately $2.6 \%$ of hypertensive patients receiving MONOPRIL. In most cases, these were isolated values which resolved despite continued therapy. In clinical trials, $0.1 \%$ of patients (two patients) were discontinued from therapy due to an elevated serum potassium. Risk factors for the development of hyperkalemia include renal insufficiency potassium-containing salt substitutes, which should be used cautiously, if at all, with MONOPRIL (Fosinopri Sodium) (see PRECAUTIONS: Drug Interactions). Impaired Liver Function: Since fosinopril is primarily metabolized by hepatic and gut wall esterases to its active moiety, fosinoprilat, patients with impaired liver function could develop elevated plasma levels of unchanged fosinopril. In a study in patients with alcoholic or biliary cirrhosis, the extent of hydrolysis was unaffected, although the rate was slowed. In these patients, the apparent Cirrhosis, the extent of hydrolysis was unaffected, although the rate was slowed. In these patients, the apparent
total body clearance of fosinoprilat was decreased and the plasma AUC approximately doubled. Surgery/ Anesthesia: In patients undergoing surgery or during anesthesia with agents that produce hypotension fosinopril will block the angiotensin II formation that could otherwise occur secondary to compensatory renin release. Hypotension that occurs as a result of this mechanism can be corrected by volume expansion Information for Patients: Angioedema: Angioedema, including laryngeal edema, can occur with treatment with ACE inhibitors, especially following the first dose. Patients should be advised to immediately report to their physician any signs or symptoms suggesting angioedema (e.g., swelling of face, eyes, lips, tongue, larynx therapy. (See WARNINGS and ADVERSE REACTIONS.) Symptomatic Hypotension: Patients should be cautioned that lightheadedness can occur, especially during the first days of therapy, and it should be reported to tioned that lightheadedness can occur, especially during the first days of therapy, and it should be reported to physician. Patients should be told that if syncope occurs, MONOPRIL should be discontinued until the physician
has been consulted. All patients should be cautioned that inadequate fluid intake or excessive perspiration, has been consulted. All patients should be cautioned that inadequate fluid intake or vomiting can lead to an excessive fall in blood pressure, with the same consequence of
diarrhea, or volus
lightheadedness and possible syncope. Hyperkalemia: Patients should be told not to use potassium supplelightheadedness and possible syncope. Hyperkalemia: Patients should be told not to use potassium supplebe told to promptlytes containing potassium without consulting the physician. Neutropenia: Patients should neutropenia. Drug Interactions: With diuretics: Patients on diuretics, especially those with intravascular volume depletion, may occasionally experience an excessive reduction of blood pressure after initiation of therapy with MONOPRIL. The possibility of hypotensive effects with MONOPRIL can be minimized by either possible, the starting dose should be reduced and the patient should be observed closely for several hours possible, the starting dose should be reduced and the patient should be observed closely for several hours potassium supplements and potassium-sparing diuretics: MONOPRIL can attenuate potassium loss caused by thiazide diuretics. Potassium-sparing diuretics (spironolactone, amiloride, triamterene, and others) or potassium supplements can increase the risk of hyperkalemia. Therefore, if concomitant use of such agents is indicated, they should be given with caution, and the patient's serum potassium should be monitored frequently With lithium: Increased serum lithium levels and symptoms of lithium toxicity have been reported in patients receiving ACE inhibitors during therapy with lithium. These drugs should be coadministered with caution, and be increased. With antacids: In a clinical pharmacology study, coadministration of an antacid (aluminum hydroxide, magnesium hydroxide, and simethicone) with fosinopril reduced serum levels and urinary excretion of fosinoprilat as compared with fosinopril administered alone, suggesting that antacids may impair absorption

of fosinopril. Therefore, if concomitant administration of these agents is indicated, dosing should be separatec single or multiple dose pharmacokinetic interaction studies with chlorthalidone, nifedipine, propranolol hydrochiorothiazide, cimetidine, metoclopramide, propantheline, digoxin, and warfarin, the bioavailability o fosinoprilat was not altered by coadministration of fosinopril with any one of these drugs. In a study witt concomitant administration of aspirin and MONOPRIL, the bioavailability of unbound fosinoprilat was no
altered. In a pharmacokinetic interaction study with warfarin, bioavailability parameters, the degree of proteir binding, and the anticoagulant effect (measured by prothrombin time) of warfarin were not significant changed. Drug/Laboratory Test Interaction: Fosinopril may cause a false low measurement of serum digoxir levels with the Digi-Tab* RIA Kit for Digoxin. Other kits, such as the Coat-A-Count* RIA Kit, may be used when fosinopril was given in the diet to mice and rats for up to 24 months at doses up to $400 \mathrm{mg} / \mathrm{kg} / \mathrm{day}$. On body weight basis, the highest dose in mice and rats is about 250 times the maximum human dose of $80 \mathrm{mg}$

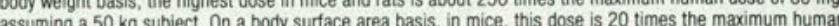
assuming a $50 \mathrm{~kg}$ subject. On a body surface area basis, in mice, this dose is 20 times the maximum humar slightly higher incidence of mesentery/omentum lipomas. Neither fosinopril nor the active fosinoprilat was mutagenic in the Ames microbial mutagen test, the mouse lymphoma forward mutation assay, or a mitotic gene conversion assay. Fosinopril was also not genotoxic in a mouse micronucleus test in vivo and a mouse bone marrow cytogenetic assay in vivo. In the Chinese hamster ovary cell cytogenetic assay, fosinopril increased the frequency of chromosomal aberrations when tested without metabolic activation at a concentration that wa without metabolic activation or at any concentration with metabolic activation. There were no adverse reproduc tive effects in male and female rats treated with 15 or $60 \mathrm{mg} / \mathrm{kg}$ daily. On a body weight basis, the high dose 0 $60 \mathrm{mg} / \mathrm{kg}$ is about 38 times the maximum recommended human dose. On a body surface area basis, this dose is 6 times the maximum recommended human dose. There was no effect on pairing time prior to mating in rat: 6 times the maximum recommended human dose. There was no effect on pairing time prior to mating in rat:
until a daily dose of $240 \mathrm{mg} / \mathrm{kg}$, a toxic dose, was given; at this dose, a slight increase in pairing time wa: until a daily dose of $240 \mathrm{mg} / \mathrm{kg}$, a toxic dose, was given; at this dose, a slight increase in pairing time wa: observed. On a body weight basis, this dose is 150 times the maximum recommended human dose. On a bod
surface area basis, this dose is 24 times the maximum recommended human dose. Pregnancy: Pregnanc Category D: See WARNINGS: Fetal/Neonatal Morbidity and Mortality. Nursing Mothers: Ingestion of $20 \mathrm{mg}$ dail? for three days resulted in detectable levels of fosinoprilat in breast milk. MONOPRIL (Fosinopril Sodium) shoule not be administered to nursing mothers. Geriatric Use: Of the total number of patients who received fosinopri in effectiveness or safety were observed between these patients and younger patients, and other reporte clinical experience has not identified differences in response between the elderly and younger patients, bu greater sensitivity of some oider individuals cannot be ruled out. In a pharmacokinetic study comparing elderi or groups in peak fosinoprilat levels or area under the plasma concentration time curve (AUC). Pediatric Use
Safety and effectiveness in children have not been established. ADVERSE REACTIONS. MONOPRIL. (Fosinopri Sodium) has been evaluated for safety in more than 1500 individuals in hypertension trials, includin approximately 450 patients treated for a year or more. Generally adverse events were mild and transient, an their frequency was not related to dose within the recommended daily dosage range. In placebo-controllen clinical trials (688 fosinopril-treated patients), the usual duration of therapy was two to three months Discontinuations due to any clinical or laboratory adverse event were 4.1 and 1.1 percent in fosinopril-treater and placebo-treated patients, respectively. The most frequent reasons $(0.4$ to $0.9 \%)$ were headache, elevate regimen, the incidence of adverse events in elderly ( $\geq 65$ years old) was similar to that seen in younger patients clinical adverse events probably or possibly related or of uncertain relationship to therapy, occurring in at leas Clinical Adverse Events in Placebo-Controlled Trials

$\begin{array}{cc}\text { MONOPRIL }(\mathrm{N}=688) & \text { Placebo }(\mathrm{N}=184) \\ \text { Incidence } & \text { Incidence }\end{array}$

\begin{tabular}{lll}
\hline Headache & $3.2(0.9)$ & 3.3 \\
Cough & $2.2(0.4)$ & 0.0 \\
Dizziness & 1.6 & 0.0 \\
Diarmea & $1.5(0.4)$ & 1.6 \\
Fatigue & $1.5(0.6)$ & 1.6 \\
Nausea/Vomiting & $1.2(0.4)$ & 0.5 \\
Sexual Dystunction & $1.0(0.1)$ & $1.1(0.5)$
\end{tabular}

Other clinical events probably or possibly related, or of uncertain relationship to therapy occurring in 0.2 to $1.0 \%$ of patients (except as noted) treated with MONOPRIL in controlled or uncontrolled clinical trials ( $\mathrm{N}=1479$ ) an less frequent, clinically significant events include (listed by body system): General: Chest pain, edema hypertensive crisis, rhythm disturbances, palpitations, hypotension, syncope, flushing, claudication Orthostatic hypotension occurred in $1.4 \%$ of patients treated with fosinopril monotherapy. Hypotension orthostatic hypotension was a cause for discontinuation of therapy in $0.1 \%$ of patients. Dermatologic. Urtican rash, photosensitivity, pruritus. Endocrine/Metabolic: Gout, decreased libido. Gastrointestinal: Pancreatitis hepatitis, dysphagia, abdominal distention, abdominal pain, flatulence, constipation, heartburn, appetite
weight change, dry mouth. Hematologic: Lymphadenopathy. Immunologic: Angioedema. Musculoskeleta. weight change, dry mouth. Hematologic: Lymphadenopathy. Immunologic: Angioedema. Musculoskeleta
Arthralgia, musculoskeletal pain, myalgia/muscle cramp. Nervous/Psychiatric: Memory disturbance, tremo confusion, mood change, paresthesia, sleep disturbance, drowsiness, vertigo. Respiratory: Bronchospasn pharyngitis, sinusitis/minitis, laryngitis/hoarseness, epistaxis. A symptom-complex of cough, bronchospasm and eosinophilia has been observed in two patients treated with fosinopril. Special Senses: Tinnitus, visio Adverse Effects Reported with ACE Inhibitors: Other medically important adverse effects reported with AC inhibitors include: Cardiac arrest; eosinophilic pneumonitis; neutropenia/agranulocytosis, pancytopenia, an mia (including hemolytic and aplastic), thrombocytopenia; acute renal failure; hepatic failure, jaundic mia (including hemolytic and aplastic), thrombocytopenia; acute renal tailure, hepatic tailure, jaund drome which may include: arthralgia/arthritis, vasculitis, serositis, myalgia, fever, rash or other dermatolog manifestations, a positive ANA, leukocytosis, eosinophilia, or an elevated ESR. Laboratory Test Abno
malities: Serum Electrolytes: Hyperkalemia, (see PRECAUTIONS); hyponatremia, (see PRECAUTIONS: Dr malities: Serum Electrolytes: Hyperkalemia, (see PRECAUTIONS); hyponatremia, (see PRECAUTIONS: Dr
Interactions, With diuretics). BUN/Serum Creatinine: Elevations, usually transient and minor, of BUN or serur creatinine have been observed. In placebo-controlled clinical trials, there were no significant differences in th number of patients experiencing increases in serum creatinine (outside the normal range or 1.33 times the pr markedly elevated blood pressure by any antihypertensive therapy can result in decreases in the glomeru filtration rate and, in turn, lead to increases in BUN or serum creatinine. (See PRECAUTIONS: Genera Hematology: In controlled trials, a mean hemoglobin decrease of $0.1 \mathrm{~g} / \mathrm{dL}$ was observed in fosinopril-treate patients. In individual patients decreases in hemoglobin or hematocrit were usually transient, small, and r associated with symptoms. No patient was discontinued from therapy due to the development of anemia. Othe Neutropenia (see WARNINGS), leukopenia and eosinophilia. Liver Function Tests: Elevations of transaminase
LDH, alkaline phosphatase and serum bilirubin have been reported. Fosinopril therapy was discontinue because of serum transaminase elevations in $0.7 \%$ of patients. In the majority of cases, the abnormalities we either present at baseline or were associated with other etiologic factors. In those cases which were possitb related to forapy.

References: 1. Hui KK, Duchin KL, Kripalani KJ, Chan D, Kramer PK, Yanagawa N. Pharmacokinetics fosinopril in patients with various degrees of renaifunction.
Cutler RE, Parmer RJ, Ford NF. Comparison of the steady-state pharmacokinetics of fosinopril, lisinopril, an enalapril in patients with chronic renal insufficiency. Clin Pharm. 1991;20:420-427. 3. Data on file, Bristo

\section{Merdगtitimin}

Bristol-Myers Squibb Hospital and Institutional Sales Group

Bristol-Myers Squibb Company

(C) 1991, Bristol-Myers Squibb Company

Boehringe
Ingelheim

Princeton, New Jersey 08543, USA
$\mathrm{J}-\mathrm{K} 72-10-$ 
the most part, become the standard item type for most examinations. Although most aspects of cognitive educational achievement can be tested by any of the item formats mentioned earlier with almost identical results, ${ }^{6}$ the multiple-choice question offers many advantages and has gained widespread acceptance. We, as educators, would do well to master it.

\section{References}

1. Coffman WE: Essay examinations, in Thorndike RL (ed): Educational measurement. Washington, DC, American Council on Education, 1971.

2. Bloom BS, et al: Taxonomy of Educational Objectives, Handbook I: Cognitive Domain. New York, David McKay Co, Inc, 1956.

3. Ebel RL: Essentials of Educational Measurement. Englewood Cliffs, NJ, Prentice-Hall, Inc, 1979, p 141.

4. Ebel RL: Maximizing test validity in fixed time limits. Educational and Psychological Measurement 1953;13:347-357.

5. Richardson MW: The relation between the difficulty and the differential validity of a test. Psychometrika 1936;1:33-49.

6. Cook DL: An investigation of three aspects of free-response and choice-type tests at the college level. Dissertation Abstracts $1955 ; 15: 1351$. 


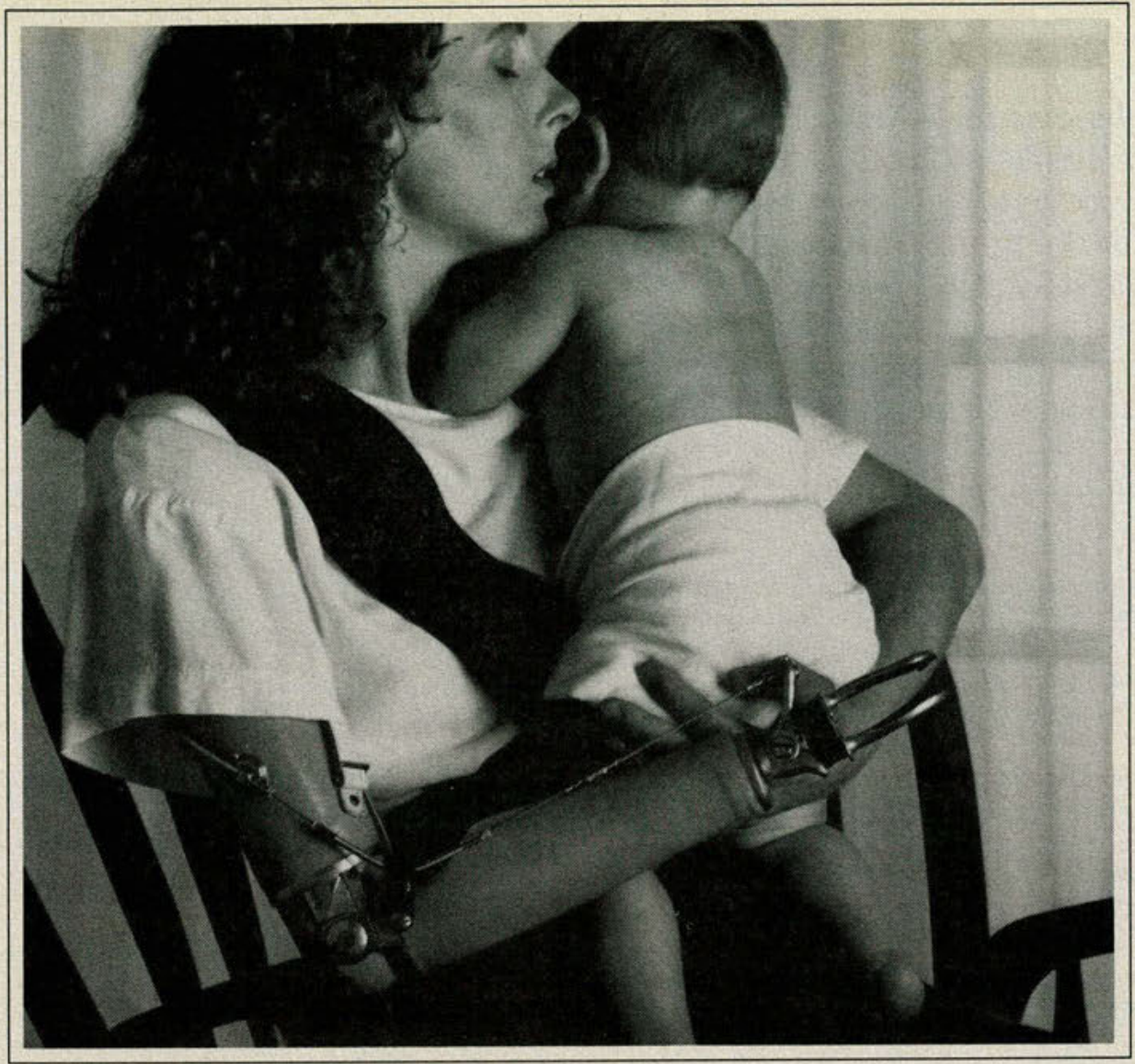

\section{She never lost her mother's touch.}

A serious accident. A serious loss.

But with help from Easter Seals this child will only feel his mother's love, his mother's tenderness.

Support Easter Seals. Give the power to overcome.

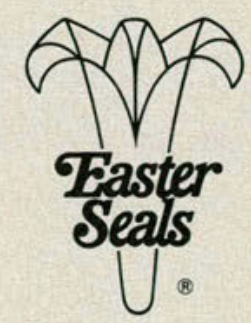

